\title{
A RELATIONSHIP BETWEEN THE DIRICHLET AND REGULARITY PROBLEMS FOR ELLIPTIC EQUATIONS
}

\author{
ZHONGWEI SHEN
}

\begin{abstract}
Let $\mathcal{L}=\operatorname{div} A \nabla$ be a real, symmetric second order elliptic operator with bounded measurable coefficients. Consider the elliptic equation $\mathcal{L} u=0$ in a bounded Lipschitz domain $\Omega$ of $\mathbb{R}^{n}$. We study the relationship between the solvability of the $L^{p}$ Dirichlet problem $(D)_{p}$ with boundary data in $L^{p}(\partial \Omega)$ and that of the $L^{q}$ regularity problem $(R)_{q}$ with boundary data in $W^{1, q}(\partial \Omega)$, where $1<p, q<\infty$. It is known that the solvability of $(R)_{p}$ implies that of $(D)_{p^{\prime}}$. In this note we show that if $(D)_{p^{\prime}}$ is solvable, then either $(R)_{p}$ is solvable or $(R)_{q}$ is not solvable for any $1<q<\infty$.
\end{abstract}

\section{Introduction}

Let $\mathcal{L}=\operatorname{div} A \nabla$ be a real, symmetric second order elliptic operator with bounded measurable coefficients. For $f \in C(\partial \Omega)$, consider the classical Dirichlet problem

$$
\begin{cases}\mathcal{L} u=0 & \text { in } \Omega \\ u=f & \text { on } \partial \Omega\end{cases}
$$

where $\Omega$ is a bounded Lipschitz domain in $\mathbb{R}^{n}, n \geq 2$. Let $\|\cdot\|_{p}$ denote the norm in $L^{p}(\partial \Omega)$ with respect to the surface measure $d \sigma$ on $\partial \Omega$. The $L^{p}$ Dirichlet problem $(D)_{p}$ is said to be solvable if the unique solution $u \in C(\bar{\Omega})$ to (1.1) satisfies the estimate $\|N(u)\|_{p} \leq C\|f\|_{p}$. Here $N(u)$ denotes the nontangential maximal function of $u$. If boundary data $f$ is in $W^{1, p}(\partial \Omega)$, i.e., the first order (tangential) derivatives of $f$ are also in $L^{p}(\partial \Omega)$, it is natural to require that the solution satisfies the condition $\|N(\nabla u)\|_{p} \leq C\|f\|_{W^{1, p}(\partial \Omega)}$. This is the so-called $L^{p}$ regularity problem. We remark that since $\nabla u$ may not be locally bounded, $N(\nabla u)$ needs to be suitably defined for weak solutions. Both the $L^{p}$ Dirichlet and regularity problems (as well as the $L^{p}$ Neumann problem) have been studied extensively. We refer the reader to the monograph $[\mathbf{K}]$ by Kenig for a survey of results as well as a list of open problems.

The purpose of this note is to study the relationship between the Dirichlet problem and the regularity problem. It is well known that the solvability of the $L^{p}$ regularity problem implies that of the $L^{p^{\prime}}$ Dirichlet problem (see e.g. [KP2]), where $p^{\prime}=p /(p-1)$ denotes the dual exponent of $p$. The converse is also true in the case of Laplace's equation $\Delta u=0$

Key words and phrases. Elliptic Equation; Dirichlet Problem; Regularity Problem.

Recieved by the editors June 17, 2006.

The author is supported in part by the NSF (DMS-0500257). 
on Lipschitz domains [ $\mathbf{V}]$. However whether the converse holds for a general second order elliptic operator with nonsmooth coefficients remains open. In this note we obtain a partial result.

Main Theorem. Let $\mathcal{L}$ be a real, symmetric second order elliptic operator of divergence form with bounded measurable coefficients. Let $1<p<\infty$ and $\Omega$ be a bounded Lipschitz domain. Suppose that the $L^{p^{\prime}}$ Dirichlet problem for $\mathcal{L}$ on $\Omega$ is solvable. Then either the $L^{p}$ regularity problem $(R)_{p}$ is solvable or $(R)_{q}$ is not solvable for any $1<q<\infty$.

We remark that for Laplace's equation in a Lipschitz domain $\Omega$, the Dirichlet problem $(D)_{p^{\prime}}$ and the regularity problem $(R)_{p}$ are solvable for $1<p<2+\varepsilon$, where $\varepsilon>0$ depends on $\Omega[\mathbf{D 1}, \mathbf{D 2}, \mathbf{J K}, \mathbf{V}]$. If $\Omega$ is a $C^{1}$ domain, $(D)_{p}$ and $(R)_{p}$ are solvable for any $1<p<\infty$ $\left[\right.$ FJR]. However, for a general second order elliptic operator $\mathcal{L},(D)_{p}$ (thus $(R)_{p^{\prime}}$ ) may not be solvable for any $1<p<\infty$, even if the coefficients of $\mathcal{L}$ are continuous and $\Omega$ is smooth. Furthermore, it is known that the Dirichlet problem $(D)_{p^{\prime}}$ for $\mathcal{L}$ on $\Omega$ is solvable if and only if the $\mathcal{L}$-harmonic measure is a $B_{p}$ weight with respect to the surface measure on $\partial \Omega$. We refer the reader to $[\mathbf{K}]$ for references on these and other deep results on the solvability of the $L^{p}$ Dirichlet problem. Concerning the $L^{p}$ regularity problem for general second order elliptic operators, we mention that the study was initiated by Kenig and Pipher in [KP1, KP2]. In particular it was proved in [KP1] that the solvability of $(R)_{p}$ implies that of $(R)_{q}$ for all $1<q<p+\varepsilon$. This fact is used in the proof of the main theorem.

Our main theorem will be proved in two steps. First we establish a weak reverse Hölder estimate,

$$
\left(\frac{1}{\left|I_{r}\right|} \int_{I_{r}}|N(\nabla u)|^{p} d \sigma\right)^{1 / p} \leq \frac{C}{\left|I_{6 r}\right|} \int_{I_{6 r}}|N(\nabla u)| d \sigma
$$

where $I_{r}$ is a surface cube on $\partial \Omega$, and $u$ is a weak solution of $\mathcal{L} u=0$ in $\Omega$ whose boundary data vanishes on $I_{6 r}$. This is done by using the comparison principle and properties of $\mathcal{L}$-harmonic measures (see Theorem 2.9). It worths pointing out that for (1.2) to hold, one only needs to assume that $(D)_{p^{\prime}}$ is solvable.

The second step of the proof of the main theorem relies on a real variable argument. It uses a maximal dyadic cube decomposition on $\partial \Omega$ and the reverse Hölder estimate (1.2) to establish a good- $\lambda$ type inequality (see Lemma 3.4). It is here that one needs to assume the solvability of $(R)_{q}$ for some $q<p$. Motivated by $[\mathbf{C P}]$ (also see $[\mathbf{W}]$ ), this approach of combining the Calderón-Zygmund decomposition with the reserve Hölder estimates was developed in $[\mathbf{S 1}, \mathbf{S 2}, \mathbf{S 3}]$ to study the $L^{p}$ bounds of Riesz transforms associated with operator $\mathcal{L}$, and the $L^{p}$ estimates for elliptic systems and higher order elliptic equations on Lipschitz domains. We remark that a similar method was used simultaneously and independently with different motivation by Auscher, Coulhon, Duong and Hofmann [ACDH, A] in the study of Riesz transforms on manifold as well as elliptic operators with complex coefficients. 


\section{A weak reverse Hölder inequality}

Throughout this note, we will use $\Omega$ to denote a bounded Lipschitz domain in $\mathbb{R}^{n}$. For $P \in \partial \Omega$, let

$$
\Gamma(P)=\Gamma_{\alpha}(P)=\{x \in \Omega: \quad|x-P|<(1+\alpha) \delta(x)\},
$$

where $\alpha>0$ is sufficiently large and $\delta(x)=\operatorname{dist}(x, \partial \Omega)$. We define

$$
N(u)(P)=\sup \left\{\left(\frac{1}{\left|B\left(x, \frac{1}{2} \delta(x)\right)\right|} \int_{B\left(x, \frac{1}{2} \delta(x)\right)}|u(y)|^{2} d y\right)^{1 / 2}: x \in \Gamma_{\alpha}(P)\right\},
$$

for any $u \in L_{\text {loc }}^{2}(\Omega)$. This is a variant of the usual nontangential maximal function $(u)^{*}$, which is defined by

$$
(u)^{*}(P)=\sup \left\{|u(x)|: x \in \Gamma_{\alpha}(P)\right\} .
$$

It is known that if $\mathcal{L} u=0$ in $\Omega$, then $\|N(u)\|_{p}$ and $\left\|(u)^{*}\right\|_{p}$ are equivalent for any $0<p \leq$ $\infty$.

Assume $0 \in \partial \Omega$ and

$$
\Omega \cap B\left(0, r_{0}\right)=\left\{\left(x^{\prime}, x_{n}\right) \in \mathbb{R}^{n}: x_{n}>\psi\left(x^{\prime}\right)\right\} \cap B\left(0, r_{0}\right),
$$

where $B\left(0, r_{0}\right)$ denotes the ball centered at 0 with radius $r_{0}$ and $\psi: \mathbb{R}^{n-1} \rightarrow \mathbb{R}$ is a Lipschitz function, $\psi(0)=0$. For $r>0$, we let

$$
\begin{aligned}
& I_{r}=\left\{\left(x^{\prime}, \psi\left(x^{\prime}\right)\right):\left|x_{1}\right|<r, \ldots,\left|x_{n-1}\right|<r\right\}, \\
& D_{r}=\left\{\left(x^{\prime}, x_{n}\right) \in \mathbb{R}^{n}:\left|x_{1}\right|<r, \ldots,\left|x_{n-1}\right|<r, \psi\left(x^{\prime}\right)<x_{n}<\psi\left(x^{\prime}\right)+r\right\} .
\end{aligned}
$$

Note that if $0<r<c r_{0}$, then $D_{r} \subset \Omega$ and $\partial D_{r} \cap \partial \Omega=I_{r}$.

Lemma 2.5. Let $u \in C(\bar{\Omega})$ be a weak solution of $\mathcal{L} u=0$ in $\Omega$. Suppose that $u=0$ on $I_{5 r}$ for some $0<r<c r_{0}$. Then for any $x \in D_{2 r}$,

$$
|u(x)| \leq C \cdot \frac{G(x, z)}{G\left(A_{r}, z\right)}\left\{\frac{1}{r^{n}} \int_{D_{4 r}}|u(y)|^{2} d y\right\}^{1 / 2},
$$

where $A_{r}=\left(0, \frac{r}{2}\right), z \in \Omega \backslash D_{10 r}$, and $G(x, y)$ denotes the Green function for $\mathcal{L}$ on $\Omega$.

Proof. Write $u=u_{1}-u_{2}$ on $D_{3 r}$, where $u_{1}, u_{2}$ are nonnegative weak solutions on Lipschitz domain $D_{3 r}$ with boundary values $u_{+}=\max (u, 0)$ and $u_{-}=\max (-u, 0)$ on $\partial D_{3 r}$ respectively. By the comparison principle for nonnegative weak solutions $[\mathbf{C F M S}]$, we have

$$
u_{j}(x) \leq C \cdot \frac{G(x, z)}{G\left(A_{r}, z\right)} \cdot u_{j}\left(A_{r}\right) \leq C \cdot \frac{G(x, z)}{G\left(A_{r}, z\right)} \cdot \max _{\partial D_{3 r}}\left|u_{j}\right|, \quad j=1,2,
$$

where $x \in D_{2 r}$ and $z \in \Omega \backslash D_{10 r}$. It follows that

$$
\begin{aligned}
|u(x)| & \leq\left|u_{1}(x)\right|+\left|u_{2}(x)\right| \leq C \cdot \frac{G(x, z)}{G\left(A_{r}, z\right)} \cdot \max _{\partial D_{3 r}}|u| \\
& \leq C \cdot \frac{G(x, z)}{G\left(A_{r}, z\right)}\left\{\frac{1}{r^{n}} \int_{D_{4 r}}|u(y)|^{2} d y\right\}^{1 / 2},
\end{aligned}
$$

where we have used the boundary $L^{\infty}$ estimate in the last step. The proof is finished. 
Theorem 2.9. Suppose $1<p<\infty$ and $(D)_{p^{\prime}}$ is solvable for operator $\mathcal{L}$ on $\Omega$. Let $u \in C(\bar{\Omega})$ be a weak solution of $\mathcal{L} u=0$ in $\Omega$. If $u=0$ on $I_{6 r}$ for some $0<r<c r_{0}$, then

$$
\left(\frac{1}{\left|I_{r}\right|} \int_{I_{r}}|N(\nabla u)|^{p} d \sigma\right)^{1 / p} \leq \frac{C}{\left|I_{6 r}\right|} \int_{I_{6 r}}|N(\nabla u)| d \sigma
$$

where $\left|I_{r}\right|$ denotes the surface measure of $I_{r}$.

Proof. We begin by observing that for any $P \in I_{r}$,

$$
N(\nabla u)(P) \leq C\left\{\left(\frac{u}{\delta}\right)_{20, r}^{*}(P)+\frac{1}{\left|I_{6 r}\right|} \int_{I_{6 r}} N(\nabla u) d \sigma\right\}
$$

where $\left(\frac{u}{\delta}\right)_{20, r}^{*}(P)=\sup \left\{\frac{|u(x)|}{\delta(x)}:|x-P| \leq c r\right.$ and $\left.x \in \Gamma_{20 \alpha}(P)\right\}$. To see (2.11), we note that if $x \in \Gamma_{\alpha}(P)$ and $|x-P| \geq c_{1} r$ for some $P \in I_{r}$, then

$$
\left(\frac{1}{\left|B\left(x, \frac{1}{2} \delta(x)\right)\right|} \int_{B\left(x, \frac{1}{2} \delta(x)\right)}|\nabla u(y)|^{2} d y\right)^{1 / 2} \leq \frac{C}{\left|I_{6 r}\right|} \int_{I_{6 r}} N(\nabla u) d \sigma .
$$

Indeed, if the left side of (2.12) is greater than $\lambda$, then

$$
\left|\left\{P \in I_{6 r}: N(\nabla u)(P)>c_{2} \lambda\right\}\right| \geq c_{2}\left|I_{6 r}\right| .
$$

This may be seen by subdividing $B\left(x, \frac{1}{2} \delta(x)\right)$. On the other hand, if $x \in \Gamma_{\alpha}(P)$ and $|x-P|<c_{1} r$ for some $P \in I_{r}$, the left side of (2.12) is bounded by

$$
C\left(\frac{1}{\left|B\left(x, \frac{3}{4} \delta(x)\right)\right|} \int_{B\left(x, \frac{3}{4} \delta(x)\right)}\left|\frac{u(y)}{\delta(y)}\right|^{2} d y\right)^{1 / 2} \leq C\left(\frac{u}{\delta}\right)_{20, r}^{*}(P) .
$$

This follows from the Cacciopoli inequality.

To estimate $\left(\frac{u}{\delta}\right)_{20, r}^{*}$ on $I_{r}$, we use Lemma 2.5. This gives us

$$
\left(\frac{u}{\delta}\right)_{20, r}^{*}(P) \leq \frac{C}{G\left(A_{r}, z\right)}\left(\frac{1}{r^{n}} \int_{D_{4 r}}|u(y)|^{2} d y\right)^{1 / 2} \cdot\left(\frac{G(\cdot, z)}{\delta(\cdot)}\right)_{20, r}^{*}(P)
$$

for any $P \in I_{r}$. Now, fix $z \in \Omega \backslash D_{10 r}$. Let $\omega=\omega^{z}$ denote the $\mathcal{L}$-harmonic measure on $\partial \Omega$, evaluated at $z$. Also, for $x=\left(x^{\prime}, x_{n}\right) \in D_{5 r}$, let $\hat{x}=\left(x^{\prime}, \psi\left(x^{\prime}\right)\right) \in \partial \Omega$ and $S(\hat{x}, t)=B(\hat{x}, t) \cap \partial \Omega$. Since

$$
\frac{G(z, x)}{\delta(x)} \approx \frac{\omega(S(\hat{x}, \delta(x))}{\delta(x)^{n-1}}
$$


for $x \in D_{2 r}$ and $\omega$ is a doubling measure [CMFS], we have

$$
\left(\frac{G(z, \cdot)}{\delta(\cdot)}\right)_{20, r}^{*}(P) \leq C M_{\sigma, r}(\omega)(P)
$$

where

$$
M_{\sigma, r}(\omega)(P)=\sup \left\{\frac{\omega^{z}(S(P, t))}{t^{n-1}}: 0<t<r\right\}
$$

is a localized Hardy-Littlewood maximal function of $\omega$ with respect to the surface measure.

Finally, since $(D)_{p^{\prime}}$ is solvable for $\mathcal{L}$ on $\Omega, \omega$ satisfies the Reverse Hölder inequality,

$$
\left(\frac{1}{|S|} \int_{S}\left|\frac{d \omega}{d \sigma}\right|^{p} d \sigma\right)^{1 / p} \leq \frac{C \omega(S)}{|S|},
$$

for any surface ball $S=B(P, t) \cap \partial \Omega$ on $\partial \Omega$. This, together with (2.14)-(2.16) as well as the $L^{p}$ boundedness of the Hardy-Littlewood maximal operator, yields

$$
\left(\frac{1}{\left|I_{r}\right|} \int_{I_{r}}\left|\left(\frac{u}{\delta}\right)_{20, r}^{*}\right|^{p} d \sigma\right)^{1 / p} \leq \frac{C}{r}\left(\frac{1}{r^{n}} \int_{D_{4 r}}|u(y)|^{2} d y\right)^{1 / 2} .
$$

To finish the proof, we note that

$$
\begin{aligned}
& \frac{1}{r}\left(\frac{1}{r^{n}} \int_{D_{4 r}}|u|^{2} d y\right)^{1 / 2} \leq C\left(\frac{1}{r^{n}} \int_{D_{4 r}}|\nabla u|^{2} d y\right)^{1 / 2} \\
& \leq \frac{C}{r^{n}} \int_{D_{5 r}}|\nabla u| d y \leq \frac{C}{r^{n-1}} \int_{I_{6 r}} N(\nabla u) d \sigma .
\end{aligned}
$$

We point out that we have used Poincaré inequality for the first inequality in (2.20) and some well known properties of weak solutions for the second. Also, the third inequality in (2.20) follows from

$$
\int_{I_{6 r}} N(\nabla u) d \sigma \geq \frac{C}{r} \int_{D_{6 r}}\left\{\frac{1}{\delta(x)^{n}} \int_{B\left(x, \frac{1}{2} \delta(x)\right)}|\nabla u(y)| d y\right\} d x
$$

and the Fubini's theorem. The desired reverse Hölder estimate (2.10) now follows by combining (2.11), (2.19) and (2.20).

\section{The proof of the Main Theorem}

Let $1<p<\infty$. Suppose that the Dirichlet problem $(D)_{p^{\prime}}$ is solvable for operator $\mathcal{L}$ on Lipschitz domain $\Omega$. Also assume that the regularity problem $(R)_{q}$ is solvable for some $1<q<p$. We will show that $(R)_{p}$ is solvable. Let $f \in W^{1, p}(\partial \Omega) \cap C(\partial \Omega)$ and $u \in C(\bar{\Omega})$ be the unique weak solution of the classical Dirichlet problem (1.1). We need to prove that

$$
\|N(\nabla u)\|_{p} \leq C\left\|\nabla_{t} f\right\|_{p},
$$


where $\nabla_{t} f$ denotes the first order tangential derivatives of $f$.

To this end, we fix $P_{0} \in \partial \Omega$. By translation and rotation, we may assume that $P_{0}$ is the origin and (2.3) holds. Let $D=\left\{\left(x^{\prime}, x_{n}\right) \in \mathbb{R}^{n}: x_{n}>\psi\left(x^{\prime}\right)\right\}$ and $D_{r}, I_{r}$ be defined as in (2.4). Define the map $\Phi: \partial D \rightarrow \mathbb{R}^{n-1}$ by $\Phi\left(x^{\prime}, \psi\left(x^{\prime}\right)\right)=x^{\prime}$. We say that $Q \subset \partial D$ is a "cube" of $\partial D$ if $\Phi(Q)$ is a cube of $\mathbb{R}^{n-1}$. The dyadic subcubes of a cube on $\partial D$ are defined similarly. We will use $\rho Q$ for $\Phi^{-1}[\rho \Phi(Q)]$. If $Q$ is a cube on $\partial D$, we define a localized Hardy-Littlewood maximal function $M_{Q}$ by

$$
M_{Q}(f)(P)=\sup _{\substack{P \in Q^{\prime} \\ \text { cube } Q^{\prime} \subset Q}} \frac{1}{\left|Q^{\prime}\right|} \int_{Q^{\prime}}|f| d \sigma .
$$

For $\lambda>0$ and $0<r<c r_{0}$, let

$$
E(\lambda)=\left\{P \in I_{r}: M_{I_{2 r}}\left(|N(\nabla u)|^{q}\right)(P)>\lambda\right\} .
$$

Lemma 3.4. Let $1<q<p<\infty$. Suppose that $(D)_{p^{\prime}}$ and $(R)_{q}$ are solvable. There exist positive constants $\varepsilon, \gamma, C_{0}$ depending only on $p, q, n, \mathcal{L}$ and $\Omega$ such that

$$
|E(A \lambda)| \leq \varepsilon|E(\lambda)|+\left|\left\{P \in I_{r}: M_{I_{2 r}}\left(\left|\nabla_{t} f\right|^{q}\right)(P)>\gamma \lambda\right\}\right|
$$

for all $\lambda \geq \lambda_{0}$, where $A=1 /(2 \varepsilon)^{q / p}$ and

$$
\lambda_{0}=\frac{C_{0}}{\left|I_{2 r}\right|} \int_{I_{2 r}}|N(\nabla u)|^{q} d \sigma
$$

Proof. Let $\varepsilon \in(0,1)$ be a small constant to be determined. By the weak $(1,1)$ estimate of the Hardy-Littlewood maximal function, we have

$$
|E(\lambda)| \leq \frac{C}{\lambda} \int_{I_{2 r}}|N(\nabla u)|^{q} d \sigma
$$

where $C$ depends only on $n$ and $\|\nabla \psi\|_{\infty}$. It follows that $|E(\lambda)|<\varepsilon\left|I_{r}\right|$ if $\lambda \geq \lambda_{0}$, where $\lambda_{0}$ is given by (3.6) with a large $C_{0}$.

Next we perform a Calderón-Zygmund decomposition on $E(\lambda)$ as a relative open subset of $I_{r}$. This produces a collection of disjoint dyadic subcubes $\left\{Q_{k}\right\}$ of $I_{r}$ such that $E(\lambda)=$ $\bigcup_{k} Q_{k}$ and each $Q_{k}$ is maximal. We may assume that $\varepsilon$ is sufficiently small so that $32 Q_{k} \subset I_{2 r}$. We claim that it is possible to choose constants $\varepsilon, \gamma, C_{0}$ so that if $Q_{k}$ is a cube with the property

$$
\left\{P \in Q_{k}: M_{I_{2 r}}\left(\left|\nabla_{t} f\right|^{q}\right)(P) \leq \gamma \lambda\right\} \neq \emptyset,
$$

then

$$
\left|E(A \lambda) \cap Q_{k}\right| \leq \varepsilon\left|Q_{k}\right| .
$$


From this, estimate (3.5) follows by summation.

To establish (3.9), we first observe that

$$
M_{I_{2 r}}\left(|N(\nabla u)|^{q}\right)(P) \leq \max \left(M_{2 Q_{k}}\left(|N(\nabla u)|^{q}\right)(P), C_{1} \lambda\right)
$$

for any $P \in Q_{k}$, where $C_{1}$ depends only on $n$ and $\|\nabla \psi\|_{\infty}$. This is because $Q_{k}$ is maximal and so $3 Q_{k} \nsubseteq E(\lambda)$. Assume $A \geq C_{1}$. It follows that

$$
\left|Q_{k} \cap E(A \lambda)\right| \leq\left|\left\{P \in Q_{k}: M_{2 Q_{k}}\left(|N(\nabla u)|^{q}\right)(P)>A \lambda\right\}\right| .
$$

Now let $v=v_{k}$ be the unique weak solution of $\mathcal{L} v=0$ in $\Omega$ with boundary data $\varphi(f-\alpha)$, where

$$
\alpha=\frac{1}{\left|17 Q_{k}\right|} \int_{17 Q_{k}} f d \sigma
$$

and $\varphi=\varphi_{k}$ is a smooth cut-off function on $\mathbb{R}^{n}$ such that $\varphi=1$ on $16 Q_{k}, \varphi=0$ on $\partial \Omega \backslash 17 Q_{k}$, and $|\nabla \varphi| \leq C / \ell_{k}$, where $\ell_{k}=\left|Q_{k}\right|^{1 /(n-1)}$. Let $\bar{p}>p$. In view of (3.11), we have

$$
\begin{aligned}
& \left|Q_{k} \cap E(A \lambda)\right| \leq\left|\left\{P \in Q_{k}: M_{2 Q_{k}}\left(|N(\nabla u-\nabla v)|^{q}\right)(P)>\frac{A \lambda}{2^{q}}\right\}\right| \\
& \quad+\left|\left\{P \in Q_{k}: M_{2 Q_{k}}\left(|N(\nabla v)|^{q}\right)(P)>\frac{A \lambda}{2^{q}}\right\}\right| \\
& \leq \frac{C}{(A \lambda)^{\bar{p} / q}} \int_{2 Q_{k}}|N(\nabla u-\nabla v)|^{\bar{p}} d \sigma+\frac{C}{A \lambda} \int_{2 Q_{k}}|N(\nabla v)|^{q} d \sigma,
\end{aligned}
$$

where we have used the weak $\left(\frac{\bar{p}}{q}, \frac{\bar{p}}{q}\right)$ and $(1,1)$ estimates for the Hardy-Littlewood maximal operator. Since $(R)_{q}$ is solvable, the second term in the right side of (3.13) is bounded by

$$
\begin{aligned}
\frac{C}{A \lambda} \int_{\partial \Omega}\left|\nabla_{t}[\varphi(f-\alpha)]\right|^{q} d \sigma & \leq \frac{C}{A \lambda} \int_{17 Q_{k}}\left|\nabla_{t} f\right|^{q} d \sigma \\
& \leq \frac{C}{A \lambda} \cdot \gamma \lambda\left|17 Q_{k}\right| \leq \frac{C \gamma}{A} \cdot\left|Q_{k}\right|,
\end{aligned}
$$

where we have used Poincaré inequality for the first inequality and (3.8) for the second.

To handle the first term on the right side of (3.13), we observe that $u-v-\alpha$ is a weak solution whose boundary data $(f-\alpha)(1-\varphi)$ vanishes on $16 Q_{k}$. Also note that the solvability of $(D)_{p^{\prime}}$ implies that $(D)_{\bar{p}^{\prime}}$ for some $\bar{p}>p$. It then follows by Theorem 2.9 that the first term on the right side of (3.13) is bounded by

$$
\begin{aligned}
& \frac{C\left|Q_{k}\right|}{(A \lambda)^{\bar{p} / q}}\left(\frac{1}{\left|12 Q_{k}\right|} \int_{12 Q_{k}}|N(\nabla u-\nabla v)| d \sigma\right)^{\bar{p}} \\
& \leq \frac{C\left|Q_{k}\right|}{(A \lambda)^{\bar{p} / q}}\left\{\left(\frac{1}{\left|12 Q_{k}\right|} \int_{12 Q_{k}}|N(\nabla u)|^{q} d \sigma\right)^{\bar{p} / q}+\left(\frac{1}{\left|12 Q_{k}\right|} \int_{12 Q_{k}}|N(\nabla v)|^{q} d \sigma\right)^{\bar{p} / q}\right\} \\
& \leq \frac{C\left|Q_{k}\right|}{(A \lambda)^{\bar{p} / q}} \cdot\left\{\lambda^{\bar{p} / q}+(\gamma \lambda)^{\bar{p} / q}\right\} \leq \frac{C}{A^{\bar{p} / q}} \cdot\left|Q_{k}\right|,
\end{aligned}
$$


where in the second inequality, we have used the solvability of $(R)_{q},(3.14)$ as well as the fact that $Q_{k}$ is maximal. This, together with (3.13) and (3.14), gives

$$
\begin{aligned}
\left|Q_{k} \cap E(A \lambda)\right| & \leq\left|Q_{k}\right|\left\{\frac{C \gamma}{A}+\frac{C}{A^{\bar{p}} / q}\right\} \\
& =\varepsilon\left|Q_{k}\right|\left\{C_{2} \gamma \varepsilon^{-\frac{q}{p}-1}+C_{2} \varepsilon^{\frac{\bar{p}}{p}-1}\right\}
\end{aligned}
$$

since $A=1 /(2 \varepsilon)^{q / p}$.

Finally, since $\bar{p}>p$, we may choose $\varepsilon>0$ so small that $C_{2} \varepsilon^{\frac{\bar{p}}{p}-1}<1 / 2$. With this $\varepsilon$ fixed, we then choose $\gamma>0$ so small that $C_{2} \gamma \varepsilon^{-\frac{q}{p}-1}<1 / 2$. The desired estimate (3.9) follows. This completes the proof.

Proof of The Main Theorem. We multiply both sides of (3.5) by $\lambda^{\frac{p}{q}-1}$ and integrate the resulting inequality in $\lambda \in\left(\lambda_{0}, \Lambda\right)$. This gives

$$
\int_{\lambda_{0}}^{\Lambda}|E(A \lambda)| \lambda^{\frac{p}{q}-1} d \lambda \leq \varepsilon \int_{\lambda_{0}}^{\Lambda}|E(\lambda)| \lambda^{\frac{p}{q}-1} d \lambda+C \int_{I_{2 r}}\left|\nabla_{t} f\right|^{p} d \sigma .
$$

Since $A^{p / q}=1 /(2 \varepsilon)$, by a change of variables, we obtain

$$
\int_{0}^{\Lambda}|E(\lambda)| \lambda^{\frac{p}{q}-1} d \lambda \leq C \lambda_{0}^{\frac{p}{q}}\left|I_{2 r}\right|+C \int_{I_{2 r}}\left|\nabla_{t} f\right|^{p} d \sigma
$$

It follows by letting $\Lambda \rightarrow \infty$ in (3.18) that

$$
\int_{I_{r}}|N(\nabla u)|^{p} d \sigma \leq C \lambda_{0}^{\frac{p}{q}}\left|I_{2 r}\right|+C \int_{I_{2 r}}\left|\nabla_{t} f\right|^{p} d \sigma
$$

In view of (3.6), we have proved that

$$
\begin{aligned}
& \left(\frac{1}{\left|I_{r}\right|} \int_{I_{r}}|N(\nabla u)|^{p} d \sigma\right)^{1 / p} \\
& \leq C\left\{\left(\frac{1}{\left|I_{2 r}\right|} \int_{I_{2 r}}|N(\nabla u)|^{q} d \sigma\right)^{1 / q}+\left(\frac{1}{\left|I_{2 r}\right|} \int_{I_{2 r}}\left|\nabla_{t} f\right|^{p} d \sigma\right)^{1 / p}\right\} .
\end{aligned}
$$

From this and the estimate $\|N(\nabla u)\|_{q} \leq C\left\|\nabla_{t} f\right\|_{q}$, we obtain $\|N(\nabla u)\|_{p} \leq C\left\|\nabla_{t} f\right\|_{p}$ by covering $\partial \Omega$ with a finite number of coordinate patches. The proof is finished. 


\section{References}

[A] P. Auscher, On necessary and sufficient conditions for $L^{p}$ estimates of Riesz transform associated to elliptic operators on $\mathbb{R}^{n}$ and related estimates, Memoirs of Amer. Math. Soc. 186 (2007).

[ACDH] P. Auscher, T. Coulhon, X.T. Duong, and S. Hofmann, Riesz transform on manifolds and heat kernel regularity, Ann. Sci. École Norm. Sup. (4) 37 (2004), no. 6, 911-957.

[CFMS] L. Caffarelli, E. Fabes, S. Mortola, and S. Salsa, Boundary behavior of nonnegative solutions of elliptic operators in divergence form, Indiana Univ. Math. J. 30 (1981), no. 4, 621-640.

[CP] L.A. Caffarelli and I. Peral, On $W^{1, p}$ estimates for elliptic equations in divergence form, Comm. Pure App. Math. 51 (1998), no. 1, 1-21.

[D1] B. Dahlberg, Estimates for harmonic measure, Arch. Rational Mech. Anal. 65 (1977), 273-288.

[D2] _ On the Poisson integral for Lipschitz and $C^{1}$ domains, Studia Math. 66 (1979), no. 1, $13-24$.

[FJR] E. Fabes, M.Jodeit Jr., and N. Riviére, Potential techniques for boundary value problems on $C^{1}$ domains, Acta Math. 141 (1978), no. 3-4, 165-186.

[JK] D. Jerison and C. Kenig, The Neumann problem on Lipschitz domains, Bull. Amer. Math. Soc. 4 (1981), no. 2, 203-207.

[K] C. Kenig, Harmonic Analysis Techniques for Second Order Elliptic Boundary Value Problems, CBMS Regional Conference Series in Math., vol. 83, AMS, Providence, RI, 1994.

[KP1] C. Kenig and J. Pipher, The Neumann problem for elliptic equations with non-smooth coefficients, Invent. Math. 113 (1993), no. 3, 447-509.

[KP2] , The Nuemann problem for elliptic equation with nonsmooth coefficients, II, Duke Math. J. 81 (1995), no. 1, 227-250.

[S1] Z. Shen, Bounds of Riesz transforms on $L^{p}$ spaces for second order elliptic operators, Ann. Inst. Fourier (Grenoble) 55 (2005), no. 1, 173-197.

[S2] - The $L^{p}$ Dirichlet problem for elliptic systems on Lipschitz domains, Math. Res. Letters 13 (2006), no. 1, 143-159.

[S3] N Necessary and sufficient conditions for the solvability of the $L^{p}$ Dirichlet problem on Lipschitz domains, Math. Ann. 336 (2006), no. 3, 672-725.

[V] G. Verchota, Layer potentials and regularity for the Dirichlet problem for Laplace's equation in Lipschitz domains, J. Funct. Anal. 59 (1984), no. 3, 572-611.

[W] L. Wang, A geometric approach to the Calderón-Zygmund estimates, Acta Math. Sinica (Engl. Ser.) 19 (2003), no. 2, 381-396.

Department of Mathematics, University of Kentucky, Lexington, KY 40506

E-mail address: shenz@ms.uky.edu 